\title{
Multiphonon capture processes in self-assembled quantum dots
}

\author{
Magnúsdóttir, Ingibjörg; Uskov, A.; Bischoff, Svend; Mørk, Jesper
}

Published in:

Summaries of Papers Presented at the Technical Digest Quantum Electronics and Laser Science Conference

Link to article, DOI:

10.1109/QELS.2001.962073

Publication date:

2001

Document Version

Publisher's PDF, also known as Version of record

Link back to DTU Orbit

Citation (APA):

Magnúsdóttir, I., Uskov, A., Bischoff, S., \& Mørk, J. (2001). Multiphonon capture processes in self-assembled quantum dots. In Summaries of Papers Presented at the Technical Digest Quantum Electronics and Laser Science Conference (pp. 206-207) https://doi.org/10.1109/QELS.2001.962073

\section{General rights}

Copyright and moral rights for the publications made accessible in the public portal are retained by the authors and/or other copyright owners and it is a condition of accessing publications that users recognise and abide by the legal requirements associated with these rights.

- Users may download and print one copy of any publication from the public portal for the purpose of private study or research.

- You may not further distribute the material or use it for any profit-making activity or commercial gain

- You may freely distribute the URL identifying the publication in the public portal 
however, have considered effects of correlations between excitons and unbound electrons or holes when a semiconductor is excited above the band gap. ${ }^{2}$ In this paper, we report the observation of a surprising correlation-induced resonance (CIR) that appears in differential transmission (DT) spectra of semiconductor quantum wells (QW) excited above the band gap. The properties of this CIR show that it is not due to biexciton formation, but instead may arise from correlations between excitons and unbound electrons or holes.

Transient, nondegenerate DT was performed on a $130 \AA$ GaAs multiple $\mathrm{QW}$. The pump pulse is tuned to $25 \mathrm{meV}$ above the exciton resonance, and is spectrally narrowed using a pulse shaper to excite electrons and holes above the band gap with well-defined initial energies. The inset to Fig. 1 shows the absorption spectrum of the sample and the spectral position of the pump. Figure 1(a) contains a reference DT spectrum for resonant excitation of HH excitons, and shows the expected biexciton-induced absorption resonance for opposite circular polarization $(\sigma+\sigma-)$ of pump and probe, but not for the same circular polarization $(\sigma+\sigma+)$. In Fig. 1(b), the DT spectrum for nondegenerate pumping also contains an induced absorption resonance; however, the energy position of this CIR is different from the biexciton and little polarization dependence is observed.

Figure 2 shows the dependence of the CIR on pump-probe delay. As can be seen in Fig. 2(a), the CIR appears almost immediately after the pump pulse, and persists for delays greater than $500 \mathrm{ps}$. Figure 2(b) shows that the bleaching at the $\mathrm{HH}$ exciton resonance $(809.0 \mathrm{~nm})$ reaches a maximum just after zero delay, while the magnitude of the CIR $(810.6 \mathrm{~nm})$ shows an initial rapid rise near zero delay but does not reach a maximum until nearly 20 ps. The fact that the CIR appears

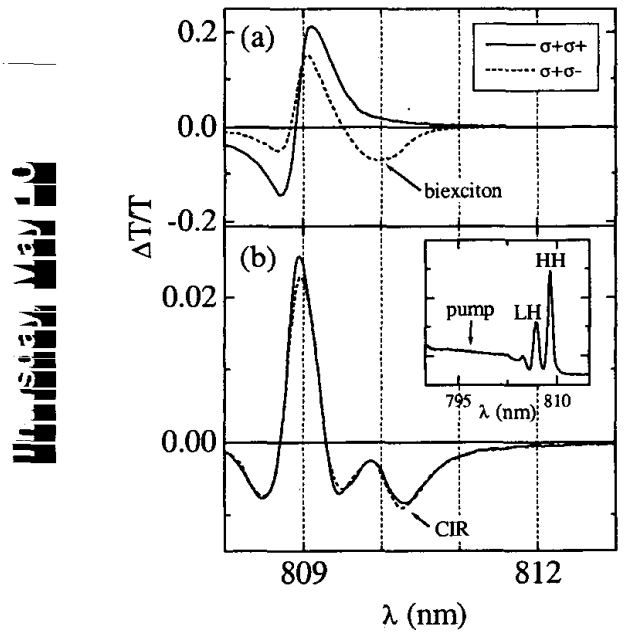

QThI8 Fig. 1. Differential transmission spectra for $\sigma+\sigma+$ (solid line) and $\sigma+\sigma-$ (dashed line) polarization of pump and probe. (a) Pump resonant with $\mathrm{HH}$ exciton transition at 2 ps pumpprobe delay. Biexciton-induced absorption ap pears for $\sigma+\sigma$-polarization. (b) Pump at $25 \mathrm{meV}$ above $\mathrm{HH}$ exciton at $10 \mathrm{ps}$ pump-probe delay. The position of the CIR is indicated. Inset shows absorption spectrum and position of nonresonant pump.
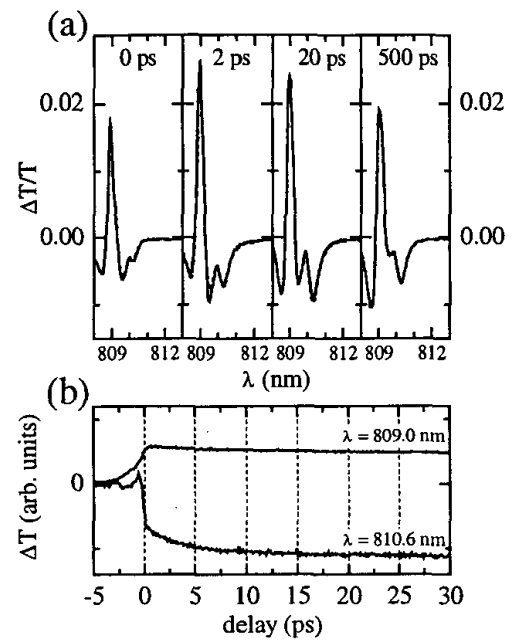

QThI8 Fig. 2. Time dependence of the differential transmission spectrum for $\sigma+\sigma+$ (solid line) and $\sigma+\sigma-$ (dashed line) pump and probe polarization. (a) Differential transmission spectra for pump-probe delays of $0,2,20$, and $500 \mathrm{ps}$. The CIR appears immediately after pumping and persists beyond 500 ps. Little polarization dependence is observed for any pump-probe delay. (b) Differential transmission at $809 \mathrm{~nm}$ (peak of the differential transmission spectrum) and at $810.6 \mathrm{~nm}$ (CIR position) versus pump-probe delay. The CIR appears immediately after pumping and reaches its maximum magnitude in $20 \mathrm{ps}$.

immediately after zero pump-probe delay indicates that it is not due to biexciton formation, because the initial electron-hole plasma excited by the pump has not had time to form excitons through phonon emission and other relaxation processes.

Another surprising feature of the CIR is shown in Fig. 3, where the DT spectrum is found to be extremely sensitive to the lattice temperature. This behavior is in sharp contrast to the biexciton resonance for resonant pumping, which shows almost no dependence on lattice temperatures over the same range. We have also investigated the dependence of the CIR on the

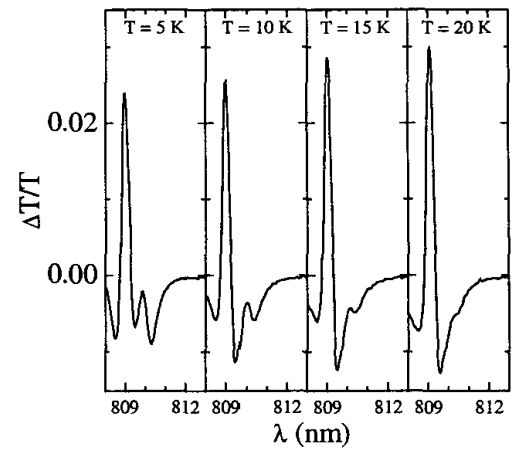

QThI8 Fig. 3. Differential transmission spectra for various lattice temperatures at pumpprobe delay of $20 \mathrm{ps}$. The CIR is strongest at low lattice temperatures and is almost unresolvable at $20 \mathrm{~K}$ pump energy. Little effect on the CIR was found for pumping at energies below, at, or above one LO-phonon energy ( $36 \mathrm{meV}$ ) above the $\mathrm{HH}$ exciton resonance.

The dependence of the CIR on polarization, pump-probe delay, and lattice temperature clearly indicates that the CIR does not reflect the formation of biexcitons. To explain these surprising results, we suggest that the CIR may arise from correlations between excitons and unbound electrons or holes.

\section{References}

1. For recent studies, see P. Kner et al., Phys. Rev. Lett. 81, 5386 (1998); C. Sieh et al., Phys. Rev. Lett. 82, 3112 (1999); Th. Östreich and L.J. Sham, Phys. Rev. Lett. 83, 3510 (1999).

2. T. Meier and S.W. Koch, Phys. Rev. B 59, 13202 (1999).

\section{QTh19}

Multiphonon capture processes in selfassembled quantum dots

I. Magnusdottir, A. Uskov, S. Bischoff, and J. Mørk, Research Center COM, Technical University of Denmark, Bldg. 345v, DK-2800 Lyngby, Denmark; Email: im@com.dtu.dk

Quantum dot (QD) lasers, with record lowthreshold currents, have now been realized in several laboratories.' Carriers in such lasers are electrically pumped into barriers around the dots. Thereafter, they are captured into the dots and relax via the QD energy levels to the lower lying lasing levels. The mechanisms for injecting electrons and holes into the QD and the time scale on which these processes occur are not fully known. These will however to a large extent determine the dynamical properties of lasers and other QD based devices. Two different mechanisms have been proposed; capture via carrier-carrier interaction (Auger processes) ${ }^{2}$ and capture via carrierphonon coupling. ${ }^{3}$ In ref. 3 , capture of a carrier from the wetting layer (WL) to the first excited state, via emission of one phonon, is found to exhibit strong resonances vs. the dot size. At resonance, the process is shown to be efficient. Furthermore, second-order elastic collisions between carriers and LO-phonons have been shown to give rise to dephasing times of a few hundred femtoseconds in $\mathrm{QDs},{ }^{4}$ in accordance with room temperature experiments. ${ }^{5}$

We investigate capture of carriers from states in the continuous part of the energy spectrum into the discrete states of self-assembled InAs/ GaAs QDs via emission of one or two phonons. We are not aware of any other investigations of two-phonon mediated capture processes in QDs, but we show that this may be an efficient capture mechanism. The phonons are assumed to be bulk GaAs LO phonons with zero dispersion. The QD was modelled by a finite confinement potential well in the effective-mass approximation.

Fig. 1 shows the capture time $(\tau)$ of an electron from a bulk region with carrier density $N_{3 D}=$ $10^{16} \mathrm{~cm}^{-3}$. From energy conservation requirements, it follows that the capture process is possible for certain intervals of QD radii, that is, there appear radius bands where $\mathrm{LO}$-phonon mediated capture can take place and gaps where it is forbidden. The bands are always larger for the two- 


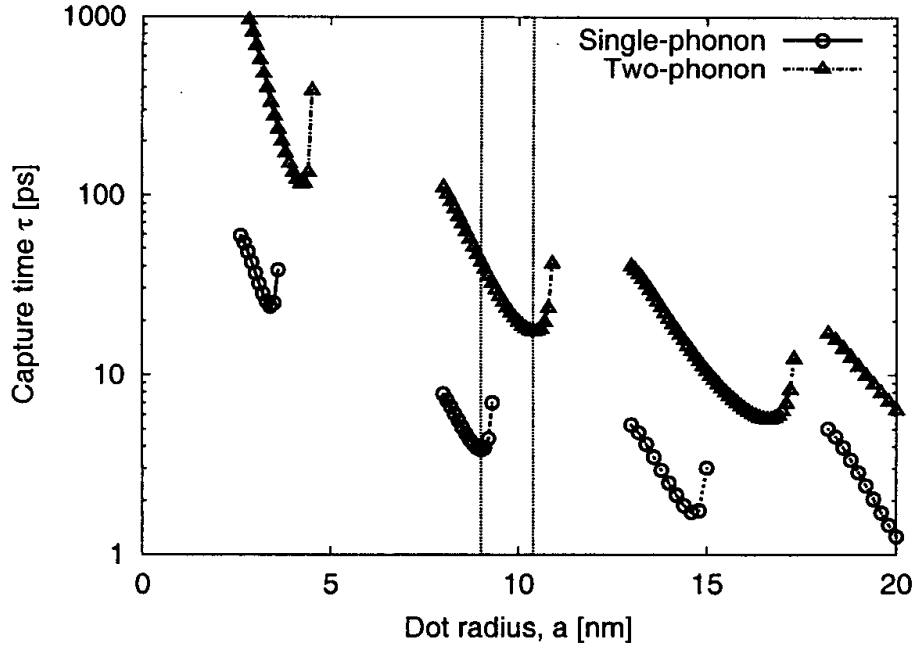

QThI9 Fig. 1. The mean single- and two-phonon capture time of an electron from the 3D continuum into the QD for the relatively low density $N_{3 \mathrm{D}}=10^{16} \mathrm{~cm}^{-3}$. The QD is assumed to be a sphere of radius a. The vertical lines indicate local minima within a radius band for the single- and two-phonon capture.

phonon capture, since carriers higher up in the band can be captured. For instance at a radius of $\approx 10 \mathrm{~nm}$, it is seen that two-phonon capture contributes where the single-phonon capture is in fact not possible. A closer look at the corresponding radius band is given in Fig. 2, where the capture time at the local minima of single- $(a=9$ $\mathrm{nm})$ and two-phonon $(\mathrm{a}=10.4 \mathrm{~nm})$ capture is calculated as a function of $N_{3 D}$. The reason for the relatively strong density depence is that only carriers from a narrow energy interval are captured and the occupation probability depends on $N_{3 D}$. The cross-over of two-phonon capture times occurs at high carrier densities where the bulk lasers start to become inverted, and is explained in terms of the Fermi-filling factors. We have also calculated the single-phonon capture time of a
WL carrier to all possible states in the QD, for $N_{2 D}$ $=10^{10} \mathrm{~cm}^{-2}$. It is found to be typically in the range $1-100 \mathrm{ps}$ and becomes even as low as a few hundred femtoseconds.

In conclusion, we have performed, to the best of our knowledge, the first calculations of twophonon capture in QDs. We find that twophonon processes may contribute efficiently to carrier injection into QDs, in situations where single-phonon processes are prohibited.

\section{References}

1. D. Bimberg, M. Grundmann, and N.N. Ledentsov, "Quantum Dot Heterostructures" (Wiley \& Sons, New York, 1999).

2. A.V. Uskov, J. McInerney, et al., "Auger carrier capture kinetics in self-assembled quantum

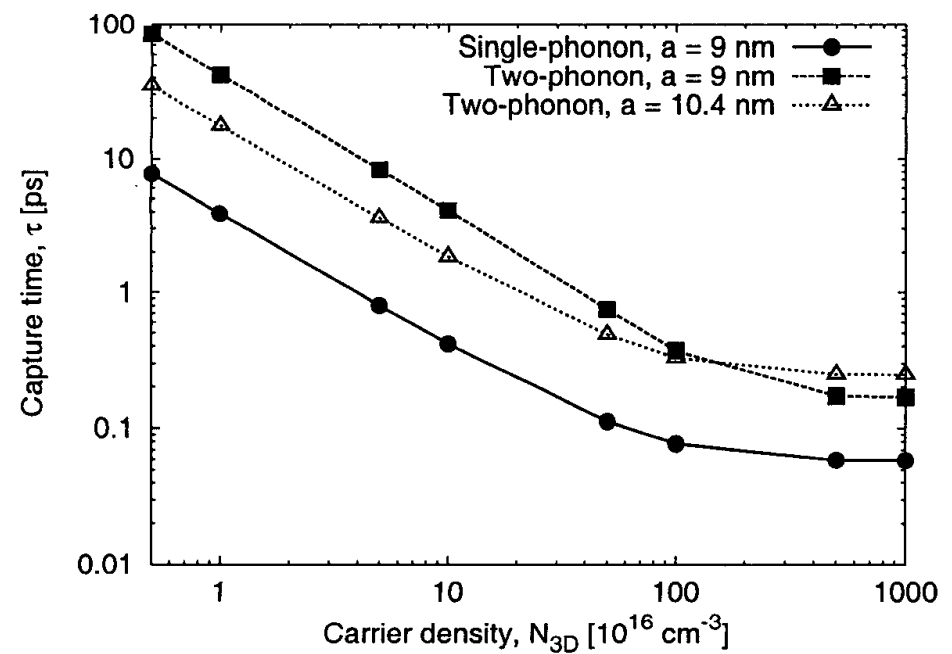

QThI9 Fig. 2. The capture time of an electron at local minima of single-phonon $(a=9 \mathrm{~nm})$ and twophonon $(\mathrm{a}=10.4 \mathrm{~nm}$ ) capture (cf. Fig. 1). At the larger radius, the single-phonon capture is forbidden. In that case, two-phonon capture is seen to contribute significantly at realistic bulk densities, well below lasing. dot structures," Appl. Phys. Lett., 72, 58-60 (1998).

3. R. Ferreira and G. Bastard, "Phonon-assisted capture and intradot Auger relaxation in quantum dots," Appl. Phys. Lett., 74, 28182820 (1999).

4. A.V. Uskov, A.-P. Jauho, et al. "Dephasing times in quantum dots due to elastic LO phonon-carrier collisions," Phys. Rev. Lett., 85, 1516-1519 (2000).

5. P. Borri, W. Langbein, et al. "Dephasing in InAs/GaAs quantum dots," Phys. Rev. B., 60, 7784-7787 (1999).

\section{QThl10}

\section{Elliptically-Polarised Gain in Semiconductor} Microcavities

P.G. Lagoudakis, P.G. Savvidis and J.J. Baumberg, Department of Physics \& Astronomy and Electronics \& Computer Science, University of Southampton, Southampton SO17 IBJ, UK; Email: lagous@phys.soton.ac.uk

A.I. Tartakovskii and M.S. Skolnick, Department of Physics, University of Sheffield, Sheffield S3 7RH, UK

J.S. Roberts, Department of Electronics and Electrical Engineering, Sheffield S1 3JD, UK

D.M. Whittaker, Toshiba Europe Research Ltd. Cambridge, CB4 4WE, UK

Semiconductor optical amplifiers have proved to be very significant for advanced telecommunications components. In traditional waveguide amplifier devices, the gain is both polarisation and wavelength dependent, and much effort has been devoted to compensating these. Such problems are removed when a circularly-symmetric vertical-cavity geometry is adopted, however the single-pass gain from such devices is normally small.

Here we report the use of semiconductor microcavities in which both electronic and photonic wavefunctions and their interaction can be specifically manipulated, to produce enormous optical amplification of ultrafast pulses. ${ }^{1,2}$ This relies on the strong coupling of excitons and photons to produce new polaritonic quasiparticles whose nonlinear interactions can be resonantly enhanced. The devices act as vertical cavity parametric amplifiers with an optical pump being efficiently downconverted into the incident signal beam. Unexpectedly the optimum gain condition is found when the pump pulse is $25 \%$ elliptically polarised. ${ }^{3}$ This state produces at least double the output compared to either a circular or a linear pump pulse. By scanning through the entire range of pump polarisations, and resolving the polarisation of the emission we are able to map out the characteristics of this phenomenon, which has also recently been seen for CW excitation. ${ }^{4}$ We propose a simple spin-dependent model for the parametric amplification, which infers the presence of a second-order stimulated scattering process that can be as strong as the first order scattering. A simulation taking this into account is in good agreement with the observations and confirms the robustness of the spin degree of freedom of polaritons. This new parametric scattering process has implications for all polarisation-dependent effects in coupled exciton-photon systems. 